\title{
A Simple and Accurate Approach to Solve the Power Flow for Balanced Islanded Microgrids
}

\author{
Faisal Mumtaz*, M. H. Syed ${ }^{\dagger}$, Mohamed Al Hosani* and H. H. Zeineldin* \\ ${ }^{*}$ Department of Electrical Engineering and Computer Science \\ Masdar Institute of Science and Technology, Abu Dhabi, UAE \\ $\dagger$ Electronics and Electrical Engineering Department, \\ University of Strathclyde, GI IXQ, Glasgow, UK
}

\begin{abstract}
Power flow studies are very important in the planning or expansion of power system. With the integration of distributed generation (DG), micro-grids are becoming attractive. So, it is important to study the power flow of micro-grids. In grid connected mode, the power flow of the system can be solved in a conventional manner. In islanded mode, the conventional method (like Gauss Seidel) cannot be applied to solve power flow analysis. Hence some modifications are required to implement the conventional Gauss Seidel method to islanded micro-grids. This paper proposes a Modified Gauss Seidel (MGS) method, which is an extension of the conventional Gauss Seidel (GS) method. The proposed method is simple, easy to implement and accurate in solving the power flow analysis for islanded microgrids. The MGS algorithm is implemented on a 6 bus test system. The results are compared against the simulations results obtained from PSCAD/EMTDC which proves the accuracy of the proposed MGS algorithm.
\end{abstract}

\section{INTRODUCTION}

Power flow studies play an important role in the planning, expansion and optimal operation of power system. Well developed power flow methods (Gauss, Gauss-Seidel, NewtonRaphson) are presented in [1]. In the recent years, scientists have focused their research in the area of grid integration of renewable energy and distributed generation (DG), which has lead to the evolution of micro-grids. These micro-grids operate either in grid connected or islanded modes. In grid connected mode, the frequency is maintained by the grid, but in islanded mode it are not constant. Several approaches have been proposed to solve power flow for islanded microgrids [2], [3]. However, these approaches are based on the assumption to classify the droop bus (the bus at which the DG is connected) either as slack, PV or PQ bus, which is invalid in case of islanded micro-grid because size of DGs are usually small and cannot act as an infinite source of power. Also in islanded micro-grid, classification of all the DGs to be PV or PQ buses is not possible [4].

The slack bus and frequency dependency issues have been recently addressed in [5]-[7], where power flow for islanded micro-grid is proposed using droop characteristics of DGs. A novel and generalized three phase power flow solved using Newton-trust region method is proposed in [5]. In [7], particleswarm technique is proposed for load flow of micro-grids. Literature suggests that the conventional load flow algorithms like
Gauss and Gauss Seidel (GS) are valid only for conventional power system and cannot be implemented to islanded microgrids [5], [7].

In this paper, a simple and accurate approach is proposed that is a modification of the conventional power flow method (Gauss Seidel) to solve the power flow problem for islanded micro-grids. The method is validated by comparing the results obtained with the simulation results obtained using time domain PSCAD/EMTDC model.

\section{System MODELING}

\section{A. Load Modeling}

The active and reactive load demand can be expressed as exponential functions of voltage and frequency [5].

$$
\begin{gathered}
P_{L k}=P_{L_{k o}}\left(\frac{V_{k}}{V_{o}}\right)^{\alpha}\left(1+K_{p}\left(\omega-\omega_{o}\right)\right), \\
Q_{L_{k}}=Q_{L_{k} o}\left(\frac{V_{k}}{V_{o}}\right)^{\beta}\left(1+K_{q}\left(\omega-\omega_{o}\right)\right),
\end{gathered}
$$

where $V_{o}$ is the nominal voltage; $P_{L_{o}}$ and $Q_{L_{o}}$ are the active and reactive power corresponding to the nominal operating voltage, respectively; $\left(\omega-\omega_{o}\right)$ is the deviation in the angular frequency. $K_{p}$ and $K_{q}$ are the frequency sensitivity parameters [8], [9]. Exponent values ( $\alpha$ and $\beta$ ) for different of loads are given in Table I.

TABLE I. LOAD TYPES AND EXPONENT VALUES [8], [10]

\begin{tabular}{lcc}
\hline Load Type $\left(L_{T}\right)$ & $\alpha$ & $\beta$ \\
\hline Constant Power (KP) & 0.00 & 0.00 \\
Constant Current (KC) & 1.00 & 1.00 \\
Constant Impedance (KI) & 2.00 & 2.00 \\
Residential (R) & 0.92 & 4.04 \\
Commercial (C) & 1.51 & 3.40 \\
Industrial (I) & 0.18 & 6.00 \\
Typical (T) & 0.92 & 1.00 \\
\hline
\end{tabular}

\section{B. Y bus Modeling}

As mentioned above, the system frequency is not constant in islanded micro-grids, and thus it affects the line reactance 
and as a result $Y_{\text {bus }}$ is not constant but a function of the system frequency given by (3).

$$
\vec{Y}^{\text {bus }}(\omega)=\left[\begin{array}{cccc}
\vec{Y}_{11}(\omega) & \vec{Y}_{12}(\omega) & \ldots & \vec{Y}_{1 N}(\omega) \\
\vec{Y}_{21}(\omega) & \vec{Y}_{22}(\omega) & \ldots & \vec{Y}_{3 N}(\omega) \\
\vdots & \vdots & \ddots & \vdots \\
\vec{Y}_{N 1}(\omega) & \overrightarrow{(Y)_{N 2}(\omega)} & \ldots & \vec{Y}_{N N}(\omega)
\end{array}\right]
$$

where $\vec{Y}_{k n}(\omega)$ is the admittance between bus $k$ and $n$.

\section{Distributed Generation (DG) Modeling}

In grid connected mode, DGs are usually modeled as PV or PQ buses. In islanded mode, since there is no slack bus, it is impossible to model all the DGs as PV or PQ buses. So DGs in an islanded micro-grids are modeled as droop buses [5]. The complex power delivered to the bus can be represented by

$$
S=P+\jmath Q,
$$

where $P$ and $Q$ are the active and reactive power delivered to the bus, respectively, and are given by [12]

$$
\left\{\begin{array}{l}
P=\frac{E V}{Z} \cos (\theta-\phi)-\frac{V^{2}}{Z} \cos (\theta) \\
Q=\frac{E V}{Z} \sin (\theta-\phi)-\frac{V^{2}}{Z} \sin (\theta)
\end{array}\right.
$$

where $E$ is the magnitude of the inverter output voltage, $\phi$ is the power angle, and $Z$ and $\theta$ are the magnitude and the phase angle of $\vec{Z}$, respectively. For an inverter based DG, its output impedance can be assumed to be inductive [13]. Assuming the output impedance of DG to be inductive, the above equation can be written as

$$
\left\{\begin{array}{l}
P=\frac{E V}{X} \sin (\phi) \\
Q=\frac{E V \cos (\phi)-V^{2}}{X}
\end{array}\right.
$$

where $X$ is the output impedance of the DG. From (6), it can be observed that for a small value of $\phi$, the active power is dependent upon the power angle $\phi$ and the reactive power is dependent upon the voltage amplitude difference. Based on the above equation the most commonly adopted droop equations for a DG can be expressed as [14], [15]

$$
\begin{aligned}
& \omega=\omega_{o}-m_{p} P_{G}, \\
& V=V_{o}-n_{q} Q_{G},
\end{aligned}
$$

where $m_{p}$ and $n_{q}$ are the frequency and voltage droop coefficients, respectively. In this paper, conventional droop equations ((7) and (8)) are considered, but the method is valid in case of resistive or complex output impedance and can be implemented by replacing conventional droop equations ((7) and (8)) with resistive or complex droop equations [12], [17].

\section{Proposed Power Flow Method}

The proposed power flow approach is based on the conventional GS method. The Modified Gauss Seidel (MGS) is developed by combining droop characteristics of DGs with the conventional GS power flow analysis.

\section{A. Types of Buses}

The well defined types of buses in the literature are slack, PV and PQ buses. The selection of bus depends upon the prespecified quantities. In this work, DG buses are classified as VF dependent (droop) buses, in which the active and reactive powers of DGs depend upon the system frequency and bus voltage. For the reference, voltage angle of bus\#1 is set to zero. In general, any bus can be a reference bus.

\section{B. Problem Formulation}

To apply GS method to an islanded micro-grid some modifications are required. Firstly, since there is no slack bus so the voltages for all the buses are variable. Secondly, the system frequency is also variable, and is required to be calculated. Also, the $Y_{\text {bus }}$ needs to be included in the iteration procedure because $Y_{b u s}$ is a function of system frequency and it will change after every iteration. Furthermore, the losses in the system need to be distributed among the DGs. To address these issues, Modified Gauss Seidel (MGS) is proposed.

\section{Modified Gauss Seidel Method}

MGS is solved in two steps. The first step is same as conventional GS method but with some modification. The step starts with the assumption of voltages to be $1 \angle 0^{\circ}$ but in this case bus\#1 (conventionally treated as slack bus) is also a variable. Furthermore, frequency is also assumed to be 1 p.u. which is calculated in second step of MGS. So there are two extra variables involved in MGS calculations. The variable vector is given by

$$
\mathbf{x}=\left[\begin{array}{ll}
\mathbf{V}^{T} & \omega
\end{array}\right]^{T} .
$$

where $\omega$ is the system frequency. $\mathbf{V}$ is the complex voltage vector (including bus\#1). The steps involved in the MGS power flow are shown in Fig. 1. To solve the first step i.e. for voltages at all buses the conventional GS voltage expression is used which is given by [1]

$$
\vec{V}_{k}^{i+1}=\frac{1}{\vec{Y}_{k k}}\left[\frac{P_{k}-\jmath Q_{k}}{\left(\vec{V}_{k}^{i}\right)^{*}}-\sum_{n=1}^{k-1} \vec{Y}_{k n} \vec{V}_{n}^{i+1}-\sum_{n=k+1}^{N} \vec{Y}_{k n} \vec{V}_{n}^{i}\right],
$$

where $\vec{V}_{k}^{i+1}$ is the voltage for iteration $i+1$ at bus $k . P_{k}$ and $Q_{k}$ are net active and reactive powers at bus $k$, respectively. For all PQ buses in the system, the above equation can be solved because both $P$ and $Q$ are known. In case of a PV bus, the reactive power is calculated using

$$
Q_{k}{ }^{i+1}=-\Im\left\{\left(\vec{V}_{k}^{i}\right)^{*}\left(\sum_{n=1}^{k-1} \vec{Y}_{k n} \vec{V}_{n}^{i+1}+\sum_{n=k}^{N} \vec{Y}_{k n} \vec{V}_{n}^{i}\right)\right\} .
$$


Once the $Q$ for the PV bus is calculated, it has to be checked for violations of its limits. Additionally, for the PV bus, once the voltage (as per (10)) is obtained, the magnitude is set back to the pre-specified value and the angle is retained. For the VF dependent, buses both active and reactive power are unknown. To calculate the active and reactive power of VF dependent buses droop equations can be used. From (7) and (8), the active and reactive power of the VF dependent bus can be calculated as

$$
\begin{aligned}
P_{G k}{ }^{i+1} & =\frac{1}{m_{p_{k}}}\left(\omega_{o}-\omega^{i}\right), \\
Q_{G_{k}}{ }^{i+1} & =\frac{1}{n_{q_{k}}}\left(V_{o}-V_{k}{ }^{i}\right) .
\end{aligned}
$$

If the active and reactive powers from (12) and 13), respectively violate the limit, they are set to their respective limit, and voltage is calculated using (10).

Once the first power flow equation is solved to obtain the voltages of all buses in the system for the $(i+1)$ iteration, the active power losses in the system are calculated. Now the second step in MGS involves frequency calculations. Since the frequency is global, all the droop buses in the micro-grid will supply active power at the same angular frequency. In an islanded micro-grid with all the DGs operating as droop based DGs, the sum of active powers of all DGs is the total active power generation of micro-grid $\left(P_{\text {sys }}\right)$ and can be represented as

$$
P_{s y s}=\sum_{k=1}^{d} P_{G k}=\sum_{k=1}^{d} \frac{1}{m_{p_{k}}}\left(\omega_{o}-\omega\right)
$$

$P_{\text {sys }}$ can be replaced by the total active power demand $\left(P_{l o a d}\right)$ plus active power loss $\left(P_{\text {loss }}\right)$. Thus $(14)$, can be modified to the following

$$
P_{\text {load }}+P_{\text {loss }}=\sum_{k=1}^{d} \frac{1}{m_{p_{k}}}\left(\omega_{o}-\omega\right)
$$

Equation (15) can be rearranged to calculate $\omega$ as

$$
\omega^{i+1}=\frac{\sum_{k=1}^{d} \frac{1}{m_{p_{k}}} \omega_{o}-\left(P_{\text {load }}^{i+1}+P_{\text {loss }}^{i+1}\right)}{\sum_{k=1}^{d} \frac{1}{m_{p_{k}}}} .
$$

Now the second power flow equation is solved for $\omega^{i+1}$ using (16). As the frequency changes in each iteration, $Y_{b u s}$ is calculated in each iteration and updated values are used to calculated the voltages. The error $(\Delta \mathbf{x})$ is evaluated, which is the difference of $\mathbf{x}^{i}$ and $\mathbf{x}^{i+1}$. If the convergence criterion is met, the system line flows are evaluated.

\section{Validation of the Proposed Methods}

To validate the proposed power flow method, the method is applied to a 6 bus test system (shown in Fig. 2). The parameters for the test systems are given in Appendix. Values of active and reactive power static droop gains are $9.4 \times 10^{-} 5 \mathrm{rad} / \mathrm{s} / \mathrm{W}$ and $0.0013 \mathrm{~V} / \mathrm{V} A R$, respectively. Three cases have been studied.

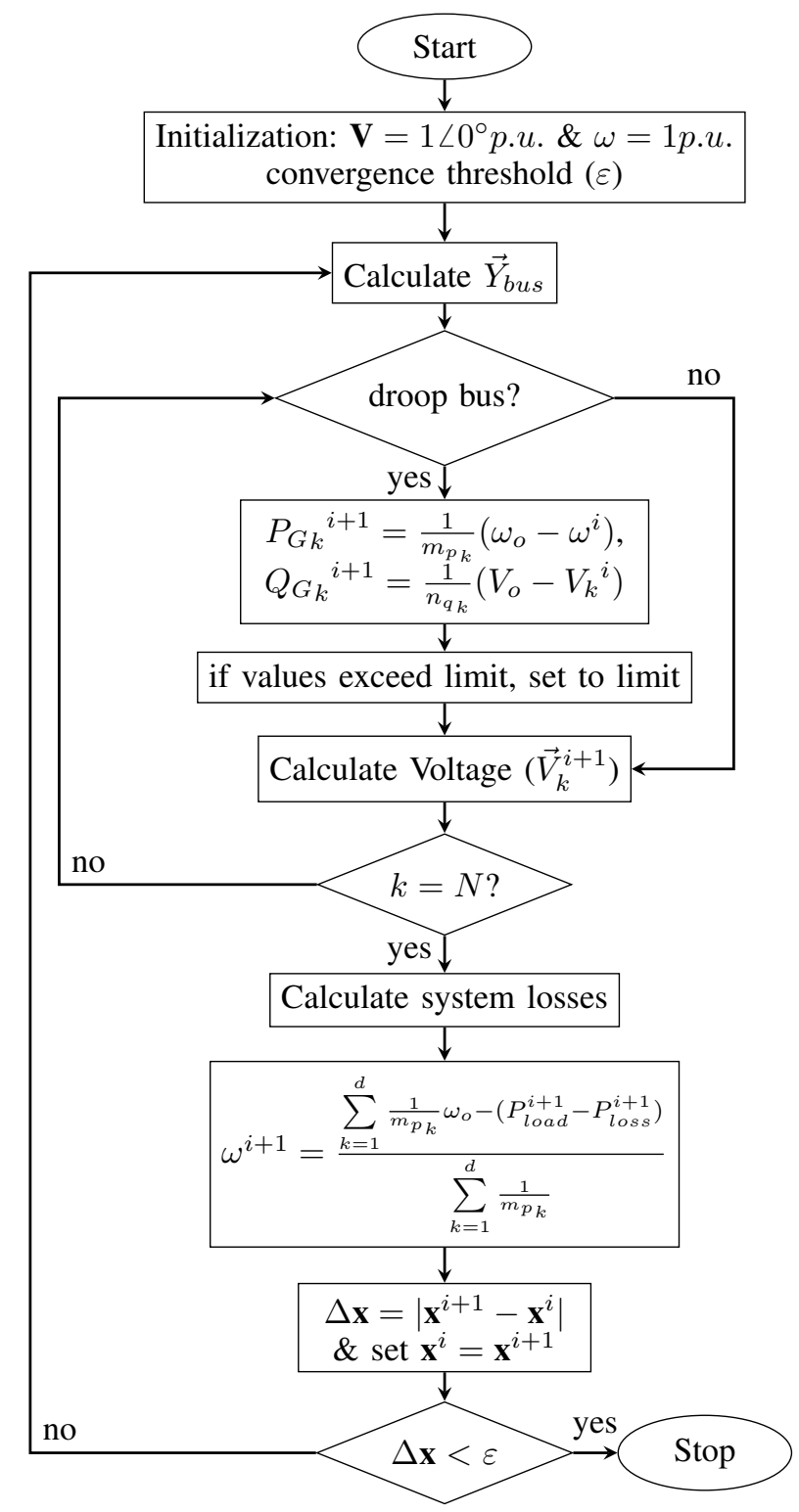

Fig. 1. MGS power flowchart

In case I, constant power load type $\left(L_{T}=K P\right)$ is considered and the results are presented in Table II. Total active power loss in this case is 0.28 p.u., and system frequency converges to 0.99903 p.u. In case II, constant impedance load type $\left(L_{T}=\right.$ $K I)$ is considered and the results are presented in Table III. Total active power loss in this case is 0.24 p.u., and system frequency converges to 0.99911 p.u. All the DGs supply same amount of active power because all DGs operate in droop mode and the droop coefficients are same for all DGs.

In case 3, MGS is validated for an islanded microgrid with mix of DGs operation. DG\#1 operates in PV mode while DG\#2 and DG\#3 operate in droop mode. The PV bus (bus\#3) supplies a fixed active power of 4.0 p.u. while regulating its voltage to 
TABlE V. Parameters For the 6-Bus Test System

\begin{tabular}{cccccc}
\hline \multicolumn{5}{c}{ Three identical DGs (10kVA 3- $\phi, 220 \mathrm{~V}(\mathrm{~L}-\mathrm{L}), 60 \mathrm{~Hz})$} \\
\hline $\mathrm{F}$ & $\mathrm{T}$ & $R_{\phi}(\Omega)$ & $X_{\phi}(m H)$ & $R_{L_{\phi}}(\Omega)$ & $Q_{L_{\phi}}(m H)$ \\
\hline 1 & 2 & 0.43 & 0.318 & 6.95 & 12.2 \\
2 & 3 & 0.15 & 1.843 & 0 & 0 \\
3 & 6 & 0.05 & 0.050 & 5.014 & 9.4 \\
4 & 1 & 0.30 & 0.350 & 0 & 0 \\
2 & 5 & 0.20 & 0.250 & 0 & 0 \\
\hline \multicolumn{7}{c}{ F=From bus, T=To bus } \\
\hline
\end{tabular}

\section{REFERENCES}

[1] J. Grainger and W. Stevenson, Power System Analysis, ser. Electrical engineering series. McGraw-Hill, 1994.

[2] H. Nikkhajoei and R. Iravani, "Steady-state model and power flow analysis of electronically-coupled distributed resource units," Power Delivery, IEEE Transactions on, vol. 22, no. 1, pp. 721-728, Jan 2007.

[3] M. Kamh and R. Iravani, "Unbalanced model and power-flow analysis of microgrids and active distribution systems," Power Delivery, IEEE Transactions on, vol. 25, no. 4, pp. 2851-2858, Oct 2010.

[4] R. Majumder, G. Ledwich, A. Ghosh, S. Chakrabarti, and F. Zare, "Droop control of converter-interfaced microsources in rural distributed generation," Power Delivery, IEEE Transactions on, vol. 25, no. 4, pp. 2768-2778, Oct 2010

[5] M. Abdelaziz, H. Farag, E. El-Saadany, and Y.-R. Mohamed, "A novel and generalized three-phase power flow algorithm for islanded microgrids using a newton trust region method," Power Systems, IEEE Transactions on, vol. 28, no. 1, pp. 190-201, Feb 2013.

[6] C. Li, S. Chaudhary, J. Vasquez, and J. Guerrero, "Power flow analysis algorithm for islanded lv microgrids including distributed generator units with droop control and virtual impedance loop," in Applied Power Electronics Conference and Exposition (APEC), 2014 Twenty-Ninth Annual IEEE, March 2014, pp. 3181-3185.

[7] A. Elrayyah, Y. Sozer, and M. Elbuluk, "A novel load-flow analysis for stable and optimized microgrid operation," Power Delivery, IEEE Transactions on, vol. 29, no. 4, pp. 1709-1717, Aug 2014.

[8] "Load representation for dynamic performance analysis [of power systems]," Power Systems, IEEE Transactions on, vol. 8, no. 2, pp 472-482, May 1993.

[9] P. Kundur, N. Balu, and M. Lauby, Power System Stability and Control, ser. Discussion Paper Series. McGraw-Hill Education, 1994. [Online]. Available: http://books.google.ae/books?id=2cbvyf8Ly4AC

[10] R. Payasi, A. Singh, and D. Singh, "Effect of voltage step constraint and load models in optimal location and size of distributed generation," in Power, Energy and Control (ICPEC), 2013 International Conference on, Feb 2013, pp. 710-716.

[11] W. Kersting, Distribution System Modeling and Analysis, Second Edition, ser. The electric power engineering series. Taylor \& Francis, 2006. [Online]. Available: http://books.google.com.sa/books?id=1R2OsUGSw_8C

[12] J. Guerrero, J. Matas, L. G. de Vicuna, M. Castilla, and J. Miret, "Decentralized control for parallel operation of distributed generation inverters using resistive output impedance," Industrial Electronics, IEEE Transactions on, vol. 54, no. 2, pp. 994-1004, April 2007.

[13] E. Rokrok and M. Golshan, "Adaptive voltage droop scheme for voltage source converters in an islanded multibus microgrid," Generation, Transmission Distribution, IET, vol. 4, no. 5, pp. 562-578, May 2010.

[14] Y. A. R. I. Mohamed and E. El-Saadany, "Adaptive decentralized droop controller to preserve power sharing stability of paralleled inverters in distributed generation microgrids," Power Electronics, IEEE Transactions on, vol. 23, no. 6, pp. 2806-2816, Nov 2008.
[15] N. Pogaku, M. Prodanovic, and T. Green, "Modeling, analysis and testing of autonomous operation of an inverter-based microgrid," Power Electronics, IEEE Transactions on, vol. 22, no. 2, pp. 613-625, March 2007.

[16] H. Bevrani and S. Shokoohi, "An intelligent droop control for simultaneous voltage and frequency regulation in islanded microgrids," Smart Grid, IEEE Transactions on, vol. 4, no. 3, pp. 1505-1513, Sept 2013.

[17] W. Yao, M. Chen, J. Matas, J. Guerrero, and Z. ming Qian, "Design and analysis of the droop control method for parallel inverters considering the impact of the complex impedance on the power sharing," Industrial Electronics, IEEE Transactions on, vol. 58, no. 2, pp. 576-588, Feb 2011.

[18] K. De Brabandere, B. Bolsens, J. Van den Keybus, A. Woyte, J. Driesen, and R. Belmans, "A voltage and frequency droop control method for parallel inverters," Power Electronics, IEEE Transactions on, vol. 22, no. 4, pp. 1107-1115, July 2007. 